\title{
Processing factors of triadimefon and triadimenol in barley brewing based on response surface methodology
}

\author{
Zhiqiang Kong a, b, 1 , Minmin $\mathrm{Li}^{\text {a, }}{ }^{\text {, }}$, Jieying Chen ${ }^{\mathrm{a}}$, Yuming Bao a , Bei Fan ${ }^{\mathrm{a}}$, \\ Frédéric Francis ${ }^{b}$, Xiaofeng Dai ${ }^{\text {a, }}{ }^{*}$ \\ ${ }^{a}$ Institute of Agro-products Processing Science and Technology, Chinese Academy of Agricultural Sciences/Key Laboratory of Agro-Products Processing/ \\ Laboratory of Agro-products Quality Safety Risk Assessment, Ministry of Agriculture, Beijing 100193, PR China \\ ${ }^{\mathrm{b}}$ Functional and Evolutionary Entomology, Gembloux Agro-Bio-Tech, University of Liége, Passage des Déportés 2, 5030 Gembloux, Belgium
}

\section{A R T I C L E I N F O}

\section{Article history:}

Received 12 October 2015

Received in revised form

14 December 2015

Accepted 14 December 2015

Available online 17 December 2015

\section{Keywords:}

Triadimefon

Behavior

Food processing

Response surface methodology

Processing factor

\begin{abstract}
A B S T R A C T
Numerous studies have focused on the effects of food-processing techniques on pesticide residues. However, it remains a challenge to ensure high-quality processing and effective pesticide removal from foods. Triadimefon (TF) is a broad-spectrum fungicide that is widely used in barley crop, and its residues are detected in its products, including beer. In this study, we investigated the dissipation kinetics of TF during fermentation mediated by two different yeast strains, Saccharomyces cerevisiae IAPPST 1401 (Y1) and CICC 1202 (Y2), and found that Y2 promoted the degradation of TF. Response surface methodology was used to optimize fermentation process variables, in order to achieve the maximum removal rate of $\mathrm{TF}$ and the minimum production of its corresponding metabolite, triadimenol (TN). Processing factors (PFs) were also evaluated during the optimized brewing process and were close to 1 for TF during the malting, milling, boiling, and cooling steps, but not in mashing and fermentation that were 0.19 and 0.13 , respectively. TF degraded to TN during brewing, and a PF value of $>1$ for TN was also observed in malting and fermentation. Our analysis concluded that beers produced using the yeasts and brewing methods we investigated are safe for human consumption.
\end{abstract}

(c) 2015 Elsevier Ltd. All rights reserved.

\section{Introduction}

Triadimefon (TF) is a registered broad-spectrum systemic sterol biosynthesis-inhibiting fungicide, which is extensively used to treat diseases and protect yield in barley (Rawlinson, Muthyalu, \& Cayley, 1982). In plants and fungi, TF is enzymatically transformed by the reduction of a carbonyl group to its corresponding alcohol, triadimenol (TN), which is also registered as a systemic fungicide with a greater pesticidal activity than that of TF (Liang et al., 2013). Previous studies have shown that TF and TN exhibit clear teratogenic effects and are harmful to the mammalian central nervous system (Menegola, Broccia, Di Renzo, Prati, \& Giavini, 2000). When fungicides are appropriately utilized, they do not cause any health- or environment-related problems. However, safety recommendations are not always followed, and undesirable residues remain in barley and found in its products, including beer

\footnotetext{
* Corresponding author.

E-mail address: daixiaofeng@caas.cn (X. Dai).

1 The authors Zhiqiang Kong and Minmin Li contributed equally to this paper.
}

(Inoue, Nagatomi, Suga, Uyama, \& Mochizuki, 2011; Navarro, Vela, \& Navarro, 2011).

Beer is one of the world's most widely consumed alcoholic beverages, which commercially produced by the controlled fermentation of wort, a liquid rich in sugars, nitrogenous compounds, sulfur compounds, and trace elements extracted from malted barley. Fermentation is the heart of brewing, in which yeast is the most important ingredient. During fermentation, a series of complex biochemical reactions occurs, producing both toxins and nutrients. As reported in previous studies, if barley contains pesticide residues, these may also be present in beer (Bajwa \& Sandhu, 2014; Hengel \& Shibamoto, 2002; Navarro, Perez, Navarro, Mena, \& Vela, 2006). Therefore, the brewing industry needs to strictly manage the risks caused by applied pesticides for ensuring consumer safety. The transfer of pesticides into beer depends on the used process and physiochemical properties of pesticides such as the water-octanol partition coefficient $\left(\mathrm{K}_{\mathrm{ow}}\right)$, solubility, and volatility (Holland, Hamilton, Ohlin, \& Skidmore, 1994; Kaushik, Satya, \& Naik, 2009; Regueiro, Lopez-Fernandez, Rial-Otero, CanchoGrande, \& Simal-Gandara, 2015). Navarro, Vela, Perez, and 
Navarro (2011) reported that flutriafol and cyproconazole (log $K_{\mathrm{ow}}$ of 2.3 and 3.1, respectively) remain in the beer after fermentation, while significantly high amounts of tebuconazole, epoxiconazole, and diniconazole ( $\log \mathrm{K}_{\mathrm{ow}}$ of 3.4-4.3) are eliminated. Hence, it is essential to investigate the fate of pesticide residues during fermentation to help the brewing industry.

In China, similar to many other countries worldwide, an increasing number of studies use the processing factors (PFs) of pesticides during food processing for the assessment of dietary risk (Aguilera, Valverde, Camacho, Boulaid, \& García-Fuentes, 2014; Boon et al., 2015; Kong et al., 2012). However, no method can simultaneously ensure the quality of processing and effectively assess the removal efficiency of pesticide residues. In addition, PF studies often fail to obtain accurate conclusions (Li et al., 2015). Hence, a statistical optimization strategy using response surface methodology (RSM) for food processing was developed to accurately track pesticide PFs. The combination of PF analysis and RSM allows pesticide residues to be monitored and controlled during food processing and strategies to be developed to enhance food safety.

This study aimed to: 1) investigate the effects of commercial brewing process (malting, milling, mashing, boiling, cooling, and fermentation) on TF and TN residues, 2) enhance the understanding on the effects of brewing process on PFs, and 3) develop an RSM approach to optimize the removal efficiency of TF and TN residues. Furthermore, beer samples of commercial brands were analyzed for TF and TN residues and evaluated by dietary exposure assessments.

\section{Materials and methods}

\subsection{Chemicals and reagents}

TF and TN analytical standards (purity $\geq 98.0 \%$ ) were acquired by Dr. Ehrenstorfer GmbH (Augsburg, Germany); commercial TF $20 \%$ emulsifiable concentrate (EC) by Jiangsu Sword Agrochemicals Co., Ltd. (Jiangsu, China); primary secondary amine (PSA) by Agela Technologies (Tianjin, China); analytical grade acetonitrile, hydrochloric acid $(\mathrm{HCl})$, anhydrous magnesium sulfate $\left(\mathrm{MgSO}_{4}\right)$, sodium chloride $(\mathrm{NaCl})$, and sodium acetate (NaAc) by Beijing Chemical Reagents (Beijing, China); spectrophotometric grade acetonitrile and acetone by Honeywell International Inc. (New Jersey, USA); and ultra-pure deionized water for Arium comfort I ultra-pure water system by Sartorius A.G. (Gottingen, Germany).

\subsection{Field trials}

Field experiments were conducted at Sheyang Farm, located in Yancheng, Jiangsu Province, China, using the barley (Hordeum vulgare L.) variety 'Yangnong 3.' Barley plants were tested and determined to be free of TF and TN before the application of target pesticides. In order to ensure adequate pesticide deposition, TF 20\% EC was applied twice $7 \mathrm{~d}$ apart at 5-fold the recommended dosage (3000 $\mathrm{g}$ active ingredient $\mathrm{ha}^{-1}$ ). Barley samples of $100 \mathrm{~kg}$ were collected $3 \mathrm{~d}$ after the last pesticide treatment, placed in polyethylene bags, and transported to the laboratory.

\subsection{In vitro assays for studying $T F$ behavior}

Two yeast strains were used to investigate the effects of fermentation on fungicide behavior: Saccharomyces cerevisiae IAPPST 1401 (Y1), which was isolated from beer in the lab; and S. cerevisiae CICC 1202 (Y2), commercially available active dry yeast. These two yeast strains were first cultured in fresh YPD medium ( $1 \%$ yeast extract, $2 \%$ peptone, and $2 \%$ glucose), grown to an optical density of 1 at $600 \mathrm{~nm}\left(\mathrm{OD}_{600}\right)$, and then placed to TF-enriched YPD liquid broth. TF-enriched YPD liquid broth without yeast was used as a control. The inoculum was added into Erlenmeyer flasks and incubated in an orbital shaker $\left(180 \mathrm{rpm}, 30^{\circ} \mathrm{C}\right)$. Three groups from each experiment were aseptically collected at $0,3,6$, and $12 \mathrm{~h}$ and 1 , $2,3,5$, and $12 \mathrm{~d}$ after inoculation. Fermentation was performed in triplicate at a TF concentration of $5.0 \mathrm{mg} \mathrm{L}^{-1}$.

\subsection{Brewing process}

A 20-kg barley sample was placed in a steep vat of water for about $40 \mathrm{~h}$. Then, barley was spread out on the floor of a germination room and rootlets began to form $3 \mathrm{~d}$ later. After germination, green malt was dried on metal racks in a kiln house at $50{ }^{\circ} \mathrm{C}$. Then, malted barley was milled using a malt mill machine (Gongda Machine Co., Ltd., Shandong, China). Milled barley was heated into $50 \mathrm{~L}$ of water at $53{ }^{\circ} \mathrm{C}$ for $70 \mathrm{~min}$, and then in a large cooking vessel, namely 'mash tun,' at around $66{ }^{\circ} \mathrm{C}$ for $80 \mathrm{~min}$. In the mash tun, grain and water created a cereal mash, in which the starch was transformed into sugar. Spent grains were filtered, and the wort was boiled at $140{ }^{\circ} \mathrm{C}$ for $90 \mathrm{~min}$ to sterilize and concentrate. During this stage, certain types of hops and carrageenan were added at different times during boiling to adjust bitterness or aroma and help with preservation. Then, the wort was quickly transferred from the brew kettle through a device to filter hops and then onto a heat exchanger for cooling. The cooled wort was saturated with air, which is essential for the growth of yeast, and transferred to a fermentation tank before adding yeast. The wort transformed to beer some days later.

\subsection{Analysis of $T F$ and $T N$ residues}

\subsubsection{Extraction and purification of barley samples}

A 5-g homogenized sample was placed into a 50-mL PTFE centrifuge tube with $3 \mathrm{~mL}$ of pure water and shaken for $1 \mathrm{~min}$. Then, $20 \mathrm{~mL}$ acetonitrile was added, and the mixture was placed on a Geno/Grinder mechanical shaker (SPEX SamplePrep, Metuchen, NJ, USA) for $3 \mathrm{~min}$ at 1200 strokes $\mathrm{min}^{-1}$. A total of $2 \mathrm{~g}$ anhydrous $\mathrm{MgSO}_{4}$ and $1 \mathrm{~g} \mathrm{NaCl}$ were added and vortexed with an $\mathrm{XW}-80 \mathrm{~A}$ Vortex (Kyova-Kirin, Tokyo, Japan) at full speed for $3 \mathrm{~min}$, and then the tube was centrifuged with a TG16-WS centrifuge (Xiangyi Centrifuge, Hunan, China) for $5 \mathrm{~min}$ at $2077 \times \mathrm{g}$. Then, $8 \mathrm{~mL}$ of the upper layer (acetonitrile) was collected in a round-bottom flask and concentrated almost to dryness using a rotary evaporator (Yarong Technologies, Shanghai, China) at $35{ }^{\circ} \mathrm{C}$. The residue was reconstituted in $2 \mathrm{~mL}$ acetonitrile and transferred to a $2.5-\mathrm{mL}$ centrifuge tube containing $50 \mathrm{mg}$ PSA and $150 \mathrm{mg}$ anhydrous $\mathrm{MgSO}_{4}$. The mixture was vortexed at full speed for $1 \mathrm{~min}$ and briefly centrifuged. Subsequently, $1 \mathrm{~mL}$ of the upper layer (acetonitrile) was filtered with a $0.22-\mathrm{mm}$ syringe filter for liquid chromatographymass spectrometry (HPLC-MS/MS) analysis.

\subsubsection{Extraction and purification of beer samples}

Beer samples of $10 \mathrm{~mL}$ each were weighed into a 50-mL PTFE centrifuge tube. Then, $600 \mu \mathrm{L}$ of $1 \mathrm{M} \mathrm{HCl}$ was added to adjust the $\mathrm{pH}$ to 2.0. Next, $10 \mathrm{~mL}$ acetonitrile was added and the mixture was placed on a Geno/Grinder mechanical shaker for $3 \mathrm{~min}$ at 1200 strokes $\mathrm{min}^{-1}$. A total of $4 \mathrm{~g}$ of anhydrous $\mathrm{MgSO}_{4}, 1 \mathrm{~g} \mathrm{NaCl}$, and $1 \mathrm{~g}$ NaAc were added and vortexed with a XW-80A Vortex at full speed for $3 \mathrm{~min}$. The tube was then centrifuged with a TG16-WS centrifuge for $5 \mathrm{~min}$ at $2077 \times \mathrm{g}$. Then, $1.5 \mathrm{~mL}$ of the upper layer (acetonitrile) was transferred to a $2.5-\mathrm{mL}$ centrifuge tube containing $50 \mathrm{mg}$ PSA and $150 \mathrm{mg}$ anhydrous $\mathrm{MgSO}_{4}$. The mixture was vortexed at full speed for $1 \mathrm{~min}$ and briefly centrifuged. Finally, $1 \mathrm{~mL}$ of the upper layer (acetonitrile) was filtered with a $0.22-\mathrm{mm}$ syringe filter for HPLC-MS analysis. 


\subsubsection{HPLC-MS/MS analytical conditions}

Chromatographic separation was performed by isocratic elution using an HPLC system equipped with an analytical column (Agilent Poroshell 120SB-C18, $4.6 \mathrm{~mm} \times 150 \mathrm{~mm}, 2.7 \mu \mathrm{m}$; Agilent Technologies, Santa Clara, CA, USA). The HPLC-MS/MS system was controlled by a MassHunter workstation (Agilent Technologies, Santa Clara, CA, USA), and LS parameters were as follows: mobile phase $A$, water containing $0.1 \%$ of formic acid (v/v) and $10 \mathrm{mmol}$ ammonium formate; mobile phase B, methanol containing $0.1 \%$ of formic acid $(\mathrm{v} / \mathrm{v})$; injection volume, $3 \mu \mathrm{L}$; and flow rate, $0.4 \mathrm{ml} \mathrm{min}^{-1}$.

A triple quadrupole MS (Agilent 6410; Agilent Technologies, Santa Clara, CA, USA) was used in the positive electrospray ionization (ESI+) mode to analyze TF and TN. An intensity of $293.7(\mathrm{~m} /$ $z$ ) was selected as the precursor ion for $\mathrm{TF}$, and its quantitative and qualitative product ions were $197.1(\mathrm{~m} / \mathrm{z})$ and $225.0(\mathrm{~m} / \mathrm{z})$, respectively. The mass transitions $\left[\mathrm{M}+\mathrm{H}^{+}\right.$of $\mathrm{TN}$ were $296.1 / 70.1$ and 296.1/99.1. The multi-reaction monitoring mode was selected as the scan mode, and MS parameters were as follows: gas flow, $10 \mathrm{~L} \mathrm{~min}^{-1}$; gas temperature, $300{ }^{\circ} \mathrm{C}$; nebulizer pressure, $40 \mathrm{psi}$; and capillary voltage, $4000 \mathrm{~V}$.

\subsection{Safety evaluation}

The impacts of TF and TN on food safety were evaluated by comparing their theoretical maximum daily intake with the maximum permissible intake (MPI). The acute reference dose (ARfD) for both TF and TN is $0.08 \mathrm{mg} \mathrm{kg}^{-1}$ body weight (BW) $\mathrm{d}^{-1}$ (JMPR, 2014). Multiplying the ARfD by $61.75 \mathrm{~kg}$, which is the average BW of an adult in China (NHFPC, 2015), produced an estimated at $4.94 \mathrm{mg}$ person ${ }^{-1} \mathrm{~d}^{-1}$ as the MPI for both TF and TN. The values of dietary exposure were calculated by multiplying the residue levels in a beer sample $\left(\mathrm{mg} \mathrm{L}^{-1}\right)$ with $0.094 \mathrm{~L}$, which is the average per capita consumption of beer per day (Cheng \& Li, 2014).

\subsection{Experimental design and data analysis}

The RSM was used to investigate the effects of process variables (temperature, time, and $\mathrm{pH}$ ) on pesticide removal rate and the concentration of the metabolite triadimenol. A Box-Behnken design (Wang et al., 2016), including 17 experimental runs formed by three variables, was used, and experimental data were fitted to a second order polynomial model as follows:

$y=b_{0}+\sum_{i} b_{i} x_{i}+\sum_{i<j} \sum_{j} b_{i j} x_{i} x_{j}+\sum_{i} b_{i i} x_{i}^{2}$,

where $x$ represents the independent variables and $y$ refers to the response that was assessed as a function of $x_{1}$ (temperature), $x_{2}$ (time), and $x_{3}(\mathrm{pH})$ and calculated as the sum of a constant $b_{0}$, the three first-order effects $\left(x_{1}, x_{2}\right.$ and $\left.x_{3}\right)$, three interaction effects $\left(x_{1} x_{2}, x_{1} x_{3}\right.$, and $\left.x_{2} x_{3}\right)$, and three second-order effects $\left(x_{1}^{2}, x_{2}^{2}\right.$, and $\left.x_{3}^{2}\right)$ in the mathematical model. Regression coefficients $(b)$ are the parameters of the model that were iteratively optimized in the subsequent study.

The specific values of three variables were identified using Derringer's desirability function, which can convert multiple responses into a single one as follows (Chen et al., 2015):

$D=\left(\prod_{i=1}^{n} d_{i}\right)^{1 / n}$

where $n$ is the number of responses, and $d i$ is the partial desirability function of each response. Data were analyzed using stepwise multiple regression in Design Expert 8.0 (Stat-Ease, Inc., Minneapolis, MN, USA) to yield equations for optimizing process variables.

The PF of one processing step is calculated as follows:

$\mathrm{PF}=\mathrm{C}_{\text {post }} / \mathrm{C}_{\mathrm{pre}}$,

where $C_{\text {post }}$ and $C_{\text {pre }}$ are post-processing and pre-processing pesticide residue concentrations $\left(\mathrm{mg} \mathrm{kg}^{-1}\right)$, respectively.

For multiple steps, the cumulative processing factor $\left(\mathrm{PF}_{\mathrm{cum}}\right)$ is calculated as follows:

$\mathrm{PF}_{\mathrm{cum}}=\mathrm{PF}_{1} \cdot \mathrm{PF}_{2} \cdots \cdots \mathrm{PF}_{\mathrm{n}}=\mathrm{C}_{\mathrm{n}} / \mathrm{C}_{0}$

where $\mathrm{PF}_{\mathrm{n}}$ is the individual processing factor for step $\mathrm{n}$, and $\mathrm{C}_{\mathrm{n}}$ and $\mathrm{C}_{0}$ are the pesticide concentrations after the nth processing step and before the initial processing step, respectively (Zhang et al., 2015).

\section{Results and discussion}

\subsection{Method validation}

The determination of $\mathrm{TF}$ and $\mathrm{TN}$ residues in barley and beer using HPLC-MS/MS was sensitive and selective, and a calibration curve of five points ranging from 0.01 to $10.0 \mathrm{mg} \mathrm{L}^{-1}$ was obtained using matrix-matched standard solutions. The correlation coefficients $\left(\mathrm{r}^{2}\right)$ of the calibration curves for TF and TN in barley and beer samples were higher than $99.8 \%$. Recovery studies were performed to validate the HPLC-MS/MS method by spiking the blank samples at three different concentration levels $(0.01,1.0$, and $10.0 \mathrm{mg} \mathrm{kg}{ }^{-1}$ ) for barley and beer and then analyzing them in quintuplicate. The results showed that the recovery of TF and TN ranged from 90.3 to $112.8 \%$ with a relative standard deviation (RSD) of $<5.2 \%$ for barley and beer (Table 1 ). These data met the requirements stipulated by the Agricultural Standards of China (NY/T 788-2004). The limit of quantification for TF and TN in barley and beer was determined to be $1.0 \mu \mathrm{g} \mathrm{kg}^{-1}$, while the limit of detection (LOD), estimated by a signal to noise ratio of 3 , was $0.3 \mu \mathrm{g} \mathrm{kg}-1$.

\subsection{Influence of different yeast strains on TF behavior}

The concentrations of TF in YPD liquid broth with or without yeast decreased with culture time, while the degradation rate of TF was faster in the presence of yeast (Fig. 1A). The results also showed that the loss of TF residues caused by Y2 (55.2\%) was higher than that caused by Y1 (48.1\%). Similar results were reported by Zhao and Wang (2012), who investigated the impact of different lactic acid bacteria on the degradation kinetics of seven different organophosphorus pesticides and found that Lactobacillus bulgaricus and Lactobacillus plantarum have a stronger impact on pesticides than Lactobacillus paracasei. Losses during fermentation may

Table 1

Average recovery and relative standard deviation of triadimefon and triadimenol in samples at different fortification levels $(n=5)$.

\begin{tabular}{|c|c|c|c|c|c|}
\hline \multirow[t]{2}{*}{ Sample } & \multirow[t]{2}{*}{ Fortification $\left(\mathrm{mg} \mathrm{kg}^{-1}\right)$} & \multicolumn{2}{|c|}{ Triadimefon } & \multicolumn{2}{|c|}{ Triadimenol } \\
\hline & & Recovery & RSD & Recovery & RSD \\
\hline \multirow[t]{3}{*}{ Barley } & 0.01 & 95.6 & 4.5 & 96.9 & 2.9 \\
\hline & 1.0 & 96.4 & 3.4 & 104.4 & 4.6 \\
\hline & 10.0 & 101.0 & 1.3 & 100.9 & 2.9 \\
\hline \multirow[t]{3}{*}{ Draft beer } & 0.01 & 90.3 & 3.8 & 91.5 & 1.2 \\
\hline & 1.0 & 101.1 & 5.2 & 99.4 & 3.4 \\
\hline & 10.0 & 107.7 & 3.8 & 112.8 & 2.4 \\
\hline
\end{tabular}



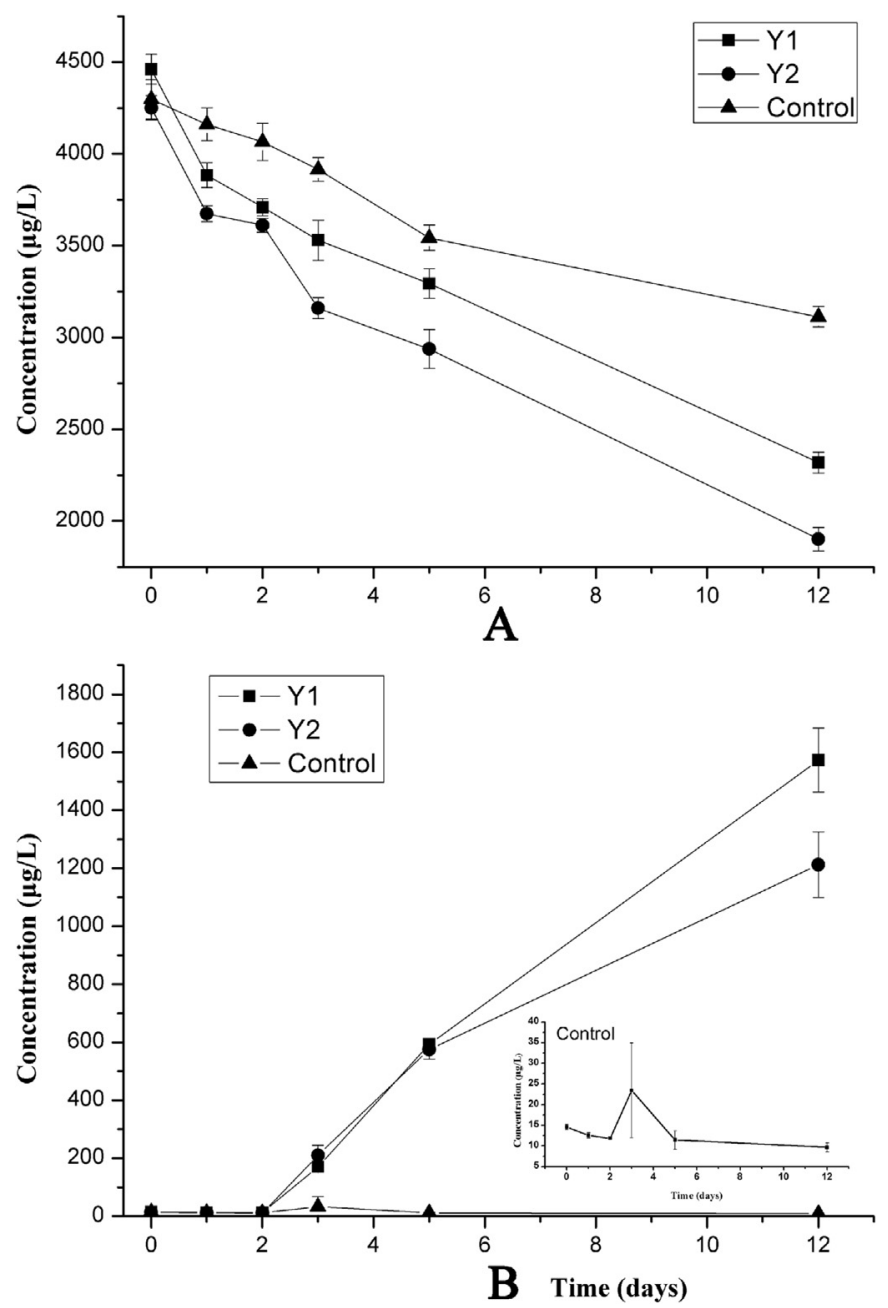

Fig. 1. Trends for triadimefon (A) and triadimenol (B) residues in YPD (1\% yeast extract, $2 \%$ peptone, and $2 \%$ glucose) liquid broth containing Saccharomyces cerevisiae IAPPST 1401 (Y1) or Saccharomyces cerevisiae CICC 1202 (Y2).

be attributed to the biotic and abiotic degradation, due to the anaerobic environment created by fermentation (Navarro, PerezLucas, Vela, \& Navarro, 2015; Regueiro et al., 2015). Additionally, the degradation of TF in YPD liquid broth without yeast may be caused by photodegradation (Nag \& Dureja, 1996). Zhang et al. (2015) reported that the exposure to sunlight could reduce the amounts of chlorpyrifos and its metabolite, 3,5,6-trichloro-2pyridinol, in grains.

As shown in Fig. 1B, the TN content in YPD liquid broth with yeast increased at a slower rate during the first 2 days and thereafter increased relatively faster as the culture time progressed. At the end of fermentation, the TN residues in YPD liquid broth with Y1 and Y2 strains were $1573.2 \mu \mathrm{g} \mathrm{L}^{-1}$ and $1212.4 \mu \mathrm{g} \mathrm{L}^{-1}$, respectively. Meanwhile, the TN content in YPD liquid broth without yeast slightly decreased during fermentation, which is probably caused by the degradation of TF to $\mathrm{TN}$, results that were consistent with those reported by Singh and Walker (2006). TF is known to be enzymatically transformed in the presence of microorganisms by the reduction of a carbonyl group to its corresponding alcohol, TN (Garrison, Avants, \& Jones, 2011; Singh, 2005). Y1 and Y2 had different effects on the removal of pesticides during fermentation; Y2 promoted more the degradation of TF residues than Y1, and as a result, it was selected as the target yeast strain in the following experiments.

\subsection{Effects of different fermentation parameters on $T F$}

The Box-Behnken design allowed the optimization of fermentation parameters, including temperature, time, and $\mathrm{pH}$, on TF. As outlined in Table 2, time ranged from 8 to $12 \mathrm{~d}$, temperature from $24^{\circ} \mathrm{C}$ to $36^{\circ} \mathrm{C}$, and $\mathrm{pH}$ from 3 to 7 . An analysis of variance (ANOVA) provided sufficient data to fit to the mathematical relationship between each response and the three variables at a confidence level of $95 \%$. None of the lack-of-fit values were significant at $p>0.05$, indicating that mathematical models fit the responses well. We obtained high coefficients of determination for the removal rate of $\mathrm{TF}(0.9876)$ and $\mathrm{TN}$ concentration (0.8953), indicating a satisfactory adjustment of the quadratic regression model with the experimental data. Accordingly, the quadratic polynomial equations for the two responses, including only statistically significant $(p<0.05)$ terms, were as follows:

Removal rate : $\mathrm{y}_{1}$

$$
\begin{aligned}
= & 51.2+1.88 x_{1}+4.88 x_{3}+3.75 x_{1} x_{3}-18.73 x_{1}^{2} \\
& -5.48 x_{2}^{2}-9.22 x_{3}^{2}
\end{aligned}
$$

TN concentration : $\mathrm{y}_{1}$

$$
\begin{aligned}
= & 1062.8+94.63 x_{3}+172.0 x_{1} x_{3} \\
& -259.78 x_{1}^{2}
\end{aligned}
$$

Therefore, temperature $\left(x_{1}\right)$ and $\mathrm{pH}\left(x_{3}\right)$, but not time $\left(x_{2}\right)$, significantly affected the removal rate and TN concentration. For the second-order effects, the squares of temperature $\left(x_{1}^{2}\right)$, time $\left(x_{2}^{2}\right)$, and $\mathrm{pH}\left(x_{3}^{2}\right)$ significantly affected the removal rate, but only the square of temperature $\left(x_{1}^{2}\right)$ significantly affected TN concentration. Analysis of interactions between terms showed that only $x_{1} x_{3}$ interaction had a significant effect on the two responses, and visible trends in $x_{1} x_{3}$ were assessed using the three-dimensional response surface plots (Fig. 2). Thereafter, the optimum variable values were identified by the over-all data and calculated by the Derringer's desirability function. The optimal conditions for achieving the best removal rate and the minimum TN level during fermentation were as follows: temperature, $27^{\circ} \mathrm{C}$; time, $12 \mathrm{~d}$; and $\mathrm{pH}$ 6. These conditions were applied in the micro-brewing process to investigate PFs of fermentation.

\subsection{Effects of brewing process steps on TF levels and processing} factors

Table 3 lists the average residues of TF and TN during the brewing process. The results indicated that $9.6 \%$ of TF in barley was degraded to TN in the field. After malting, the mean loss of TF was $13 \%$, while the concentration of TN was increased by $26 \%$, indicated that the degradation of TF to TN. The malting process includes three main stages, steeping, germination, and kilning, that involve heat treatment and biological transformation (Navarro et al., 2015), which decreases $\mathrm{TF}$ content and increases the content of its metabolite, TN. In this study, the amounts of TF and TN remained stable in barley after milling, although the milling process led to the release of enzymes and acids that may increase the rate of hydrolytic and other degradation processes in pesticide residues (Holland et al., 1994). Mashing efficiently removed $81 \%$ of TF and almost all of TN residues. TF and TN had $\log \mathrm{K}_{\mathrm{ow}}$ values of 2.3 and 3.18, respectively, and as a result, they were immobile in plant tissues and deposited in spent grains. TN was not detected in the wort after mashing $(<\mathrm{LOD})$. A number of studies have reported that the decrease in the $\mathrm{K}_{\mathrm{ow}}$ of residues increases their removal by mashing 
Table 2

Experimental values of response variables based on fermentation parameters for the response surface design.

\begin{tabular}{|c|c|c|c|c|c|c|c|c|}
\hline \multirow[t]{2}{*}{ Experimental runs } & \multicolumn{3}{|l|}{ Variables } & \multicolumn{3}{|c|}{ Symbol code } & \multirow[t]{2}{*}{ TF removal rate (\%) } & \multirow[t]{2}{*}{ Triadimenol concentration $\left(\mu \mathrm{g} \mathrm{L}^{-1}\right)$} \\
\hline & Temperature $\left({ }^{\circ} \mathrm{C}\right)$ & Time $(\mathrm{d})$ & $\mathrm{pH}$ & $\mathrm{X}_{1}$ & $\mathrm{X}_{2}$ & $\mathrm{X}_{3}$ & & \\
\hline 1 & 24 & 8 & 5 & -1 & -1 & 0 & 24 & 640 \\
\hline 2 & 36 & 8 & 5 & 1 & -1 & 0 & 30 & 760 \\
\hline 3 & 24 & 12 & 5 & -1 & 1 & 0 & 28 & 690 \\
\hline 4 & 36 & 12 & 5 & 1 & 1 & 0 & 26 & 720 \\
\hline 5 & 24 & 10 & 3 & -1 & 0 & -1 & 20 & 608 \\
\hline 6 & 36 & 10 & 3 & 1 & 0 & -1 & 18 & 540 \\
\hline 7 & 24 & 10 & 7 & -1 & 0 & 1 & 21 & 480 \\
\hline 8 & 36 & 10 & 7 & 1 & 0 & 1 & 34 & 1100 \\
\hline 9 & 30 & 8 & 3 & 0 & -1 & -1 & 32 & 860 \\
\hline 10 & 30 & 12 & 3 & 0 & 1 & -1 & 30 & 660 \\
\hline 11 & 30 & 8 & 7 & 0 & -1 & 1 & 40 & 1005 \\
\hline 12 & 30 & 12 & 7 & 0 & 1 & 1 & 44 & 840 \\
\hline 13 & 30 & 10 & 5 & 0 & 0 & 0 & 54 & 1212 \\
\hline 14 & 30 & 10 & 5 & 0 & 0 & 0 & 52 & 960 \\
\hline 15 & 30 & 10 & 5 & 0 & 0 & 0 & 50 & 1120 \\
\hline 16 & 30 & 10 & 5 & 0 & 0 & 0 & 48 & 998 \\
\hline 17 & 30 & 10 & 5 & 0 & 0 & 0 & 52 & 1024 \\
\hline
\end{tabular}

Symbol code: $\mathrm{X}_{1}, \mathrm{X}_{2}$ and $\mathrm{X}_{3}$ were coded independent variables for temperature, time, and pH, respectively.
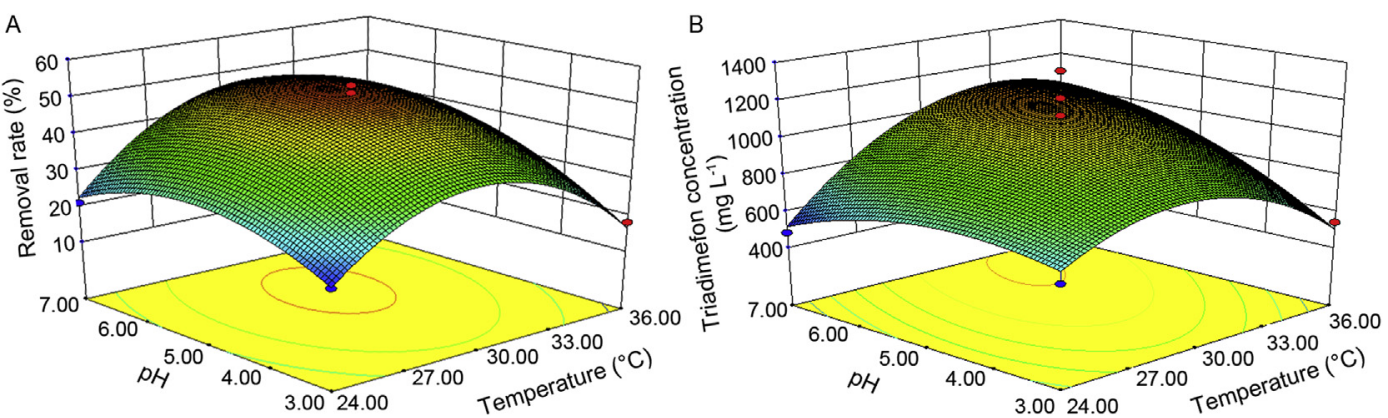

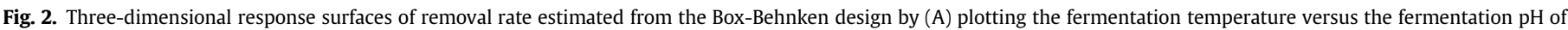
triadimenol concentration and (B) the interaction of the fermentation temperature versus the fermentation $\mathrm{pH}$.

Table 3

Amount of triadimefon and triadimenol residues $\left(\mu \mathrm{g} \mathrm{kg}^{-1}\right)$ recovered from samples after various steps in the brewing processes.

\begin{tabular}{lcl}
\hline Samples & Triadimefon $($ mean \pm SD $)$ & Triadimenol $($ mean \pm SD $)$ \\
\hline Raw barley & $14830^{\mathrm{f}} \pm 723$ & $1586^{\mathrm{e}} \pm 99$ \\
Malted barley & $12950^{\mathrm{e}} \pm 453$ & $2154^{\mathrm{d}} \pm 194$ \\
Milled barley & $12630^{\mathrm{e}} \pm 980$ & $2109^{\mathrm{d}} \pm 134$ \\
Wort & $2406^{\mathrm{c}} \pm 203$ & $<\mathrm{LOD}^{\mathrm{d}}$ \\
Spent grains & $6639^{\mathrm{d}} \pm 389$ & $1080^{\mathrm{c}} \pm 105$ \\
Hot wort & $2053^{\mathrm{bc}} \pm 212$ & $188^{\mathrm{b}} \pm 13$ \\
Cooled wort & $1902^{\mathrm{b}} \pm 118$ & $169^{\mathrm{b}} \pm 20$ \\
Beer & $254^{\mathrm{a}} \pm 27$ & $376^{\mathrm{a}} \pm 32$ \\
\hline
\end{tabular}

$\mathrm{SD}$, standard deviation; LOD, limit of detection.

$\mathrm{a}-\mathrm{f}$ Values with the different letters are significantly different $(\mathrm{p}<0.05)$.

(Inoue et al., 2011; Navarro, Vela, \& Perez, et al., 2011; Regueiro et al., 2015). Spent grains can be reused after mashing; however, $6639 \mu \mathrm{g} \mathrm{kg}^{-1} \mathrm{TF}$ and $1080 \mu \mathrm{g} \mathrm{kg}^{-1} \mathrm{TN}$ were detected after the mashing step, and their re-use was not possible. After boiling and cooling, the amount of TF in the cooled wort declined by $20.9 \%$, while TN was detectable in hot wort. During thermal processing, the loss of pesticide residues may occur through evaporation, codistillation, and thermal degradation, and metabolites may be produced by hydrolysis or pyrolysis from the parent compound (Huan, Xu, Jiang, Chen, \& Luo, 2015). When the optimized fermentation process was followed, the PFs of TF, as well as the content of TN were lower.

The PFs obtained for TF and TN are graphically represented in Fig. 3. The results showed that, except for mashing and

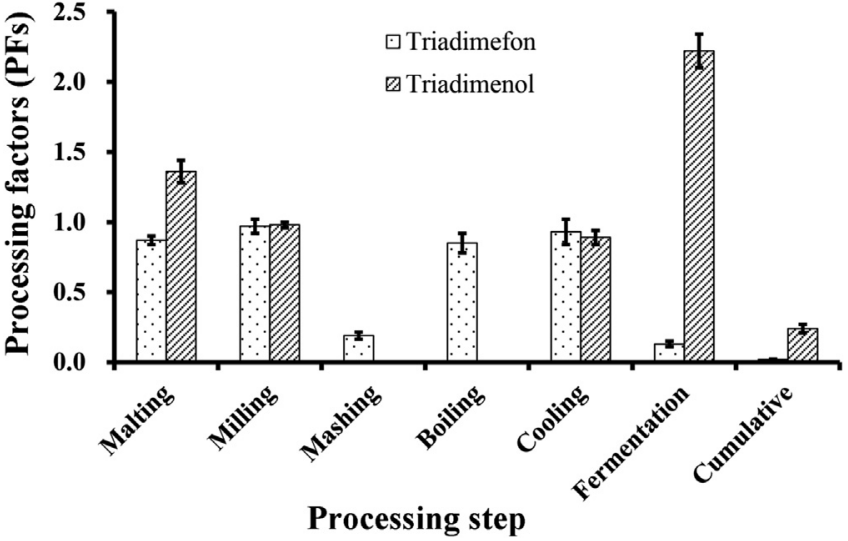

Fig. 3. Individual processing factors (PFs) and cumulative processing factors (PFcum).

fermentation, the PFs of TF were close to 1 in the malting, milling, boiling, and cooling steps during the brewing process, indicating that these steps did not affect TF residues significantly. This result may be due to the different physicochemical properties of pesticides, such as $\mathrm{K}_{\mathrm{ow}}$ and thermal stability, affect the removal of pesticide residues during food processing. TF may degrade to TN through thermolysis and biodegradation during brewing; therefore, a PF value of $>1$ for TN was also observed in malting and fermentation. $\mathrm{PF}_{\text {cum }}$ are estimated to be approximately 0.02 and 0.24 for TF and TN, respectively, after brewing. 
Table 4

Safety evaluation of commercial beer samples.

\begin{tabular}{|c|c|c|c|c|c|c|}
\hline Pesticides & No. of positive detection & \% Detection & Detection range $\left(\mathrm{mg} \mathrm{L}^{-1}\right)$ & Average conc. (mg L $\left.{ }^{-1}\right)$ & MPI (mg person ${ }^{-1}$ day $^{-1}$ ) & Dietary exposure (mg person ${ }^{-1}$ day $^{-1}$ ) \\
\hline Triadimefon & & $14.5 \%$ & $<0.06$ & 0.015 & 4.94 & $1.4 \cdot 10^{-3}$ \\
\hline Triadimenol & 5 & $9.1 \%$ & $<0.02$ & 0.007 & 4.94 & $6.6 \cdot 10^{-4}$ \\
\hline
\end{tabular}

\subsection{Monitoring and safety evaluation of commercial beer samples}

In China, the maximum residue limit (MRL) for beer is not yet established; however, the MRL of TF and TN in barley is $0.2 \mathrm{mg} \mathrm{kg}^{-1}$ (GB 2763-2014, 2014). The results showed that beer produced from barley treated twice with 5 -fold the recommended dosage of TN contained residues that exceeded the MRL value of $0.2 \mathrm{mg} \mathrm{kg}^{-1}$. To conduct a relatively accurate safety evaluation for TF and TN, a total of 55 beer samples from three different commercial brands were tested. The residue data and safety evaluation of the market samples are presented in Table 4 . The results showed that $14.5 \%$ of the samples contained TF and 9.1\% contained TN, but the dietary exposures calculated against the average concentrations were below the MPI values for barley. Therefore, beer samples were considered safe for human consumption and presented no acute toxicity hazard.

\section{Conclusions}

This study demonstrated the dissipation of TF and TN during the brewing process. Of two different yeast strains tested, S. cerevisiae CICC 1202 promoted the degradation of TF during fermentation more effectively than did S. cerevisiae IAPPST 1401. The RSM was used to optimize fermentation process variables and showed that fermentation for 12 days at $27^{\circ} \mathrm{C}$ and $\mathrm{pH} 6$ provides the maximum removal rate of TF and resulted in the minimum remaining amount of TN. The PFs of TF were close to 1 in malting, milling, boiling, and cooling, but not in mashing and fermentation, indicating that prior steps (malting, milling, boiling, and cooling) did not affect TF residues significantly. TF can degrade to TN during brewing, and a PF value of $>1$ for TN was also obtained in malting and fermentation. Overall, our analysis revealed that beer produced from barley that had been treated with fungicides and fermented using two different yeast strains was considered safe for human consumption in China.

\section{Acknowledgments}

This study was supported by the National Natural Science Foundation of China (31301477 and 31401580). We thank the University of Liège-Gembloux Agro-Bio Tech (MOE11BE1A20131371N) and more specifically the research platform AgricultureIsLife for the funding of this research project.

\section{References}

Aguilera, A., Valverde, A., Camacho, F., Boulaid, M., \& García-Fuentes, L. (2014), Household processing factors of acrinathrin, fipronil, kresoxim-methyl and pyridaben residues in green beans. Food Control, 35, 146-152.

Bajwa, U., \& Sandhu, K. S. (2014). Effect of handling and processing on pesticide residues in food-a review. Journal of Food Science and Technology, 51, 201-220.

Boon, P. E., Donkersgoed, G., Christodoulou, D., Crepet, A., D'Addezio, L., Desvignes, V., et al. (2015). Cumulative dietary exposure to a selected group of pesticides of the triazole group in different European countries according to the EFSA guidance on probabilistic modeling. Food and Chemical Toxicology, 79, 13-31.

Chen, Z., Dong, F., Li, S., Zheng, Z., Xu, Y., Xu, J., et al. (2015). Response surface methodology for the enantioseparation ofdinotefuran and its chiral metabolite in bee products andenvironmental samples by supercritical fluidchromatography/tandem mass spectrometry. Journal of Chromatography A, 1410, 181-189.
Cheng, Y., \& Li, X. (2014). China's beer consumption and its impacts on malting barley demand. Agricultural Outlook, 3, 71-73 (in Chinese).

Chinese National Health and Family Planning Commission (NHFPC). (2015). 2015 report on Chinese nutrition and chronic disease. Beijing: State Information Office.

Garrison, A. W., Avants, J. K., \& Jones, W. J. (2011). Microbial transformation of triadimefon to triadimenol in soils: selective production rates of triadimeno stereoisomers affect exposure and risk. Environmental Science and Technology, 45, 2186-2193.

GB 2763-2014. (2014). National food safety standard-maximum residue limits for pesticides in food.

Hengel, M. J., \& Shibamoto, T. (2002). Method development and fate determination of pesticide-treated hops and their subsequent usage in the production of beer. Journal of Agricultural and Food Chemistry, 50, 3412-3418.

Holland, P., Hamilton, D., Ohlin, B., \& Skidmore, M. (1994). Effects of storage and processing on pesticide residues in plant products. Pure and Applied Chemistry, $66,335-356$

Huan, Z., Xu, Z., Jiang, W., Chen, Z., \& Luo, J. (2015). Effect of Chinese traditiona cooking on eight pesticides residue during cowpea processing. Food Chemistry, $170,118-122$.

Inoue, T., Nagatomi, Y., Suga, K., Uyama, A., \& Mochizuki, N. (2011). Fate of pesticides during beer brewing. Journal of Agricultural and Food Chemistry, 59, 3857-3868.

Joint FAO/WHO Meeting on Pesticide Residues (JMPR). (2014). Acceptable daily in takes, acute reference doses, short-term and long-term dietary intakes, recommended maximum residue limits and supervised trials median residue values recorded by the 2014 meeting. Rome: FAO.

Kaushik, G., Satya, S., \& Naik, S. N. (2009). Food processing a tool to pesticide residue dissipation-a review. Food Research International, 42, 26-40.

Kong, Z., Dong, F., Xu, J., Liu, X., Zhang, C., Li, J., et al. (2012). Determination of difenoconazole residue in tomato during home canning by UPLC-MS/MS. Food Control, 23, 542-546.

Liang, H., Li, L., Qiu, J., Li, W., Yang, S., Zhou, Z., et al. (2013). Stereoselective transformation of triadimefon to metabolite triadimenol in wheat and soil under field conditions. Journal of Hazardous Materials, 260, 929-936.

Li, M., Liu, Y., Fan, B., Lu, J., He, Y., Kong, Z., et al. (2015). A chemometric processingfactor-based approach to the determination of the fates of five pesticides during apple processing. LWT Food Science and Technology, 63, 1102-1109.

Menegola, E., Broccia, M. L., Di Renzo, F., Prati, M., \& Giavini, E. (2000). In vitro teratogenic potential of two antifungal triazoles: triadimefon and triadimenol. In Vitro Cellular \& Developmental Biology-Animal, 36, 88-95.

Nag, S. K., \& Dureja, P. (1996). Phototransformation of triadimefon on glass and soil surfaces. Pesticide Science, 48, 247-252.

Navarro, S., Perez-Lucas, G., Vela, N., \& Navarro, G. (2015). Behavior of triazole fungicide residues from barley to beer. In V. R. Preedy (Ed.), Processing and impact on active components in food (pp. 525-532). San Diego, CA: Elsevier Inc.

Navarro, S., Perez, G., Navarro, G., Mena, L., \& Vela, N. (2006). Decay of dinitroaniline herbicides and organophosphorus insecticides during brewing of lager beer. Journal of Food Protection, 69, 1699-1706.

Navarro, S., Vela, N., \& Navarro, G. (2011). Fate of triazole fungicide residues during malting, mashing and boiling stages of beer making. Food Chemistry, 124, $278-284$.

Navarro, S., Vela, N., Perez, G., \& Navarro, G. (2011). Effect of sterol biosynthesisinhibiting (SBI) fungicides on the fermentation rate and quality of young ale beer. Food Chemistry, 126, 623-629.

NY/T 788-2004, Agricultural industry standards of the People's Republic of China: Guideline on pesticide residue trials.

Rawlinson, C. J., Muthyalu, G., \& Cayley, G. R. (1982). Residual effects of triadimefon in soil on powdery mildew and yield of spring barley. Plant Pathology, 31, $143-155$.

Regueiro, J., Lopez-Fernandez, O., Rial-Otero, R., Cancho-Grande, B., \& SimalGandara, J. (2015). A review on the fermentation of foods and the residues of pesticides-biotransformation of pesticides and effects on fermentation and food quality. Critical Reviews in Food Science and Nutrition, 55, 839-863.

Singh, N. (2005). Factors affecting triadimefon degradation in soils. Journal of Agricultural and Food Chemistry, 53, 70-75.

Singh, B. K., \& Walker, A. (2006). Microbial degradation of organophosphorus compounds. FEMS Microbiology Reviews, 30, 428-471.

Wang, G., Deng, Y., Xu, X., He, X., Zhao, Y., Zou, Y., et al. (2016). Optimization of air jet impingement drying of okara using response surface methodology. Food Control, 59, 743-749.

Zhang, Z., Jiang, W. W., Jian, Q., Song, W., Zheng, Z., Wang, D., et al. (2015). Changes of field incurred chlorpyrifos and its toxic metabolite residues in rice during food processing from-RAC-to-consumption. Plos One, 10, 1-13.

Zhao, X. H., \& Wang, J. (2012). A brief study on the degradation kinetics of seven organophosphorus pesticides in skimmed milk cultured with Lactobacillus spp. at $42{ }^{\circ} \mathrm{C}$. Food Chemistry, 131, 300-304. 